\title{
Expression of mRNAs and Proteins Associated with Cell-wall-loosening during Eustoma Flower Opening
}

\author{
Masaki Ochiai ${ }^{1 * *}$, Shogo Matsumoto ${ }^{1}$, Masahiro Maesaka ${ }^{1}$ and Kunio Yamada ${ }^{2 *}$ \\ ${ }^{1}$ Graduate School of Bioagricultural Sciences, Nagoya University, Nagoya 464-8601, Japan \\ ${ }^{2}$ College of Bioscience and Biotechnology, Chubu University, Kasugai 487-8501, Japan
}

Flower opening is important for floricultural crops. The mechanisms flower opening associated with the expansion of petal cells were investigated in Eustoma grandiflorum (Raf.) Shinn. Eustoma petals showed marked changes in their fresh weight, shape, and color during flower opening. Concurrently, petal cell-wall extensibility increased. This suggests that petal growth through flower opening is mainly caused by cell expansion. Expansin and xyloglucan endotransglycosylase/hydrolase $(\mathrm{XTH})$ are known as representative proteins that loosen cell walls in plants. Three expansins and one XTH gene were isolated from opening Eustoma petals. We monitored for the first changes in their protein abundance in growing petals by Western blot analysis using antibodies to specifically detect expansin or XTH. The accumulation of these proteins marked the highest amount in petals when the flower was blooming and the petals were bending outwards. Thus, we showed that expansins participate in continuous petal growth from bud to opening flower and XTH plays a role in rapid petal growth accompanied by dynamic changes in petal fresh weight and petal shape.

Key Words: gene expression, lisianthus, petal growth, Western blot, xyloglucan endotransglycosylase/hydrolase.

\section{Introduction}

The vase life of cut flowers depends on both the speed of flower opening and senescence of the petals. Although many studies have focused on senescence to improve the flower vase life (van Doorn and Woltering, 2008), the physiological study of flower opening has been reported less frequently (de Vetten and Huber, 1990; Yamada et al., 2009); therefore, revealing the mechanism of flower opening would improve the quality of ornamental flowers. Flower opening is mainly due to the expansion of petal cells. Sugar accumulation in petal cells is believed to make the chief contribution to reducing water potential and promoting water influx for cell expansion and flower opening (Evans and Reid, 1988; Ho and Nichols, 1977; Ichimura et al., 2003); however, it has been proposed that water influx and cell expansion do not occur when the cell wall of the petal remains rigid. In stems and coleoptiles, mechanical properties of the cell walls have been studied by several

Received; October 24, 2012. Accepted; November 20, 2012.

This research was supported by a Grant-in-Aid for JSPS Fellows (No. 226424) to M.O..

* Corresponding author (E-mail: yamakuni@isc.chubu.ac.jp).

** Research Fellow of the Japan Society for the Promotion of Science. analyses evaluating auxin-, gibberellin-, and acidinduced increases in extensibility (Adams et al., 1975; Carpita and Kanabus, 1988; Hohl and Schopfer, 1992; Matsukura et al., 1998; Nakamura et al., 1975; Tanimoto and Masuda, 1971; Yamamoto et al., 1970). The physical parameters and cell-wall extensibility under low $\mathrm{pH}$ have been investigated to clarify rheological processes in the elongation of cell walls (Tanimoto et al., 2000). Thus, it has been proposed that cell wall extensibility is also a growth-limiting factor for petal expansion.

Expansin and xyloglucan endotransglycosylase/ hydrolase (XTH) are candidates for cell wall-loosening proteins (Cosgrove, 2001). Expansins were the first to be identified in the wall protein fraction, which could recover acid-induced extension of heat-inactivated cell walls (McQueen-Mason et al., 1992). Expansins are believed to disrupt hydrogen bonding between cellulose microfibrils and matrix glucans (Cosgrove, 2000). Their genes belong to a multigene family, and two families recognized as $\alpha$ - and $\beta$-expansin are commonly identified from expanding and growing tissues (Cosgrove, 2001). Expansin expression has been reported in several ripening fruits (Brummel et al., 1999; Civello et al., 1999; Rose et al., 1997) and the abscission zone (Belfield et al., 2005; Sane et al., 2007).

XTHs are capable of catalyzing either the molecular 
grafting or disassembly of xyloglucan cross-links within the cellulose-xyloglucan framework (Nishitani and Tominaga, 1992; Okazawa et al., 1993). They participate in loosening and rearranging the cell walls (Rose et al., 2002), and their expressions are detected in growing tissues and ripening fruits (Catalá et al., 2000; Rose and Bennett, 1999). In petals, expansins and XTHs have been identified in several species (Gookin et al., 2003; Harada et al., 2010; Takahashi et al., 2007), and the function of $\alpha$-expansin was analyzed using a transgenic antisense approach in Petunia hybrida (Zenoni et al., 2004). The predominance of XTH activity was associated with the opening of sandersonia flowers (O'Donoghue et al., 2002) and carnation flowers (Harada et al., 2010); however, there are few reports of the protein levels of expansin and XTH during flower opening, although their mRNA expression patterns have been a focus in previous reports (Sane et al., 2007; Singh et al., 2011; Yamada et al., 2009).

Eustoma has wide variety of flower color, size, and shape. Moreover, cut Eustoma flowers, which have relatively long vase life, are produced stably throughout the year. Thus, Eustoma is one of the most important ornamental plants for cut flowers in Japan and has a great advantage for genetic analyses due to the applicable transformation technique. In this study, we clarified how expansins and XTHs are involved in flower opening of Eustoma through analyzing their mRNA expression and protein levels.

\section{Materials and Methods}

\section{Plant materials}

Flowers of Eustoma grandiflorum (Raf.) Shinn. 'Light Pink Thumb' (Fukukaen Nursery \& Bulb Co., Ltd., Aichi, Japan), which is a dwarf cultivar, were obtained from our university farm. The flower stages used for experiments are shown in Figure 1 (Stage I, bud $15 \mathrm{~mm}$ long; Stage II, bud $30 \mathrm{~mm}$ long; Stage III, bud $35 \mathrm{~mm}$ long; Stage IV, expanded bud with pinkish petals; Stage $\mathrm{V}$, opening flower and petals not bent outward; Stage VI, opening flower and petals bent outward). Petals from each flower were sampled and weighed before being frozen in liquid nitrogen.

\section{Cell wall extension analysis}

Fresh petals were cut into $5 \mathrm{~mm}$-wide strips parallel to the main vein, and then the strips were frozen at $-30^{\circ} \mathrm{C}$ and thawed at room temperature. Petal strips were secured between 2 clamps positioned $5 \mathrm{~mm}$ apart on a tensile tester KES-G1-SH (KATO TECH, Kyoto, Japan) and extended at constant $10 \mathrm{~g}$ weight at $25^{\circ} \mathrm{C}$. The distance between clamps was recorded $30 \mathrm{~min}$ after starting.

\section{Total RNA extraction}

Total RNA was isolated with TRIzol reagent (Invitrogen Corporation, Carlsbad, CA, USA) according to the instructions of the manufacturer. RNA was
A
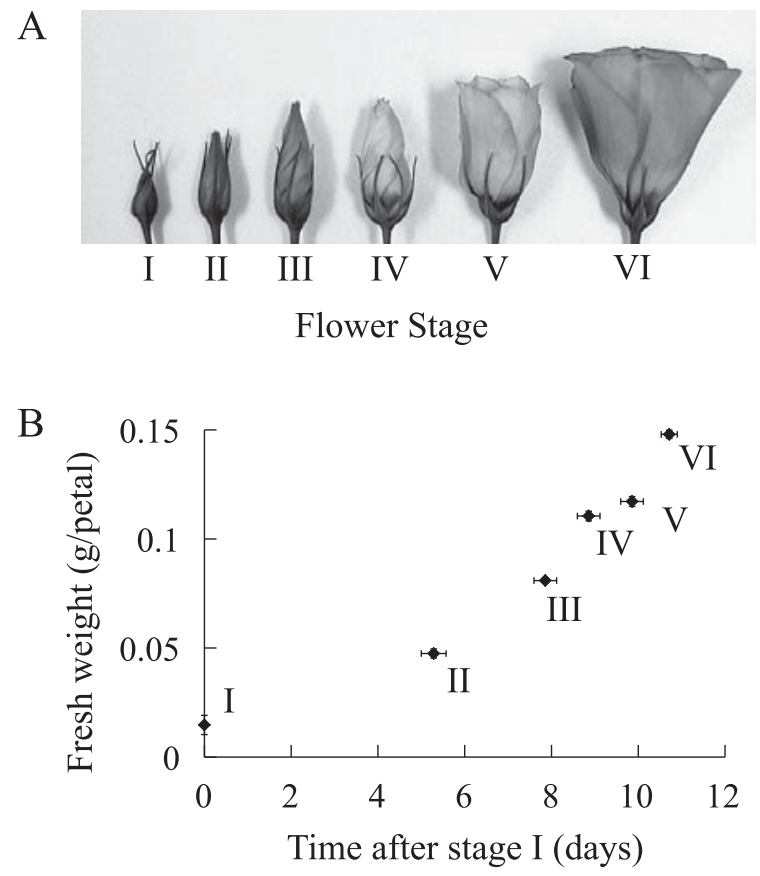

Fig. 1. Eustoma flower stages. Flowering stages of Eustoma grandiflorum (Raf.) Shinn. 'Light Pink Thumb' (A). Stage I, bud $15 \mathrm{~mm}$ long; stage II, bud $30 \mathrm{~mm}$ long; stage III, bud $35 \mathrm{~mm}$ long; stage IV, expanded bud with pinkish petals; stage V, opening flower with petals that do not bend outward; stage VI, opening flower with petals bent outward. Fresh weight of petals at each flower stage (B). Data are the means \pm SE of 19-22 independent samples. $\mathrm{X}$-axis represents the time needed to reach each flower stage after stage I. Y-axis represents the fresh weight of petals. Data are the means \pm SE of 7 independent samples.

extracted from petals at each stage, mature leaves, young leaves, stems, roots, sepals, stamens, and pistils.

\section{cDNA cloning for $\alpha$-expansins and XTHs}

Total RNA for cDNA cloning was extracted from Stage $V$ petals. First-strand cDNA was synthesized using a High Fidelity RNA PCR kit (Takara, Shiga, Japan) with an oligo dT-adaptor primer (Takara). PCR was performed with an Adapter Primer (Takara) and the forward primers that were previously designed for cDNA cloning for $\alpha$-expansin or XTH from rose petals (Takahashi et al., 2007), using the first-strand cDNA described above as the PCR template. PCR products were cloned into a pT7Blue T-vector (Novagen, Madison, WI, USA) and sequenced by a Big Dye Terminator v3.1 cycle sequence kit (Applied Biosystems, Foster City, CA, USA) using an automatic DNA sequencer (3130Avant Genetic Analyzer; Applied Biosystems). Determination of the full-length cDNAs was performed by 5'RACE using the 5'-Full RACE Core Set (Takara).

\section{Quantitative real-time $R T-P C R$}

Gene-specific mRNA quantification was performed using a SYBR ExScript RT-PCR kit (Perfect Real Time) (Takara) in a Thermal Cycler Dice Real Time System (Takara), according to the protocol provided by the 
supplier. Paralog-specific primers for each gene were mainly designed based on the $3^{\prime}$ un-translated region sequence of each cDNA. Primer pairs for each paralog were as follows: EgEXPA1 (accession no. AB762067), "CCG AGA TCT TTT GGA TCG AG" and "CTG CAG TCT CGA GTT CAT GG"; EgEXPA2 (accession no. AB762068), "TAC CTC CAC CTC CTG GAA CA" and "TTC CCT TCT GCA GGT TCT TG"; EgEXPA3 (accession no. AB762069), "GGA AGA ACC CTC ACA AGC AA" and "TCC TAA AAT TGG TTG CCT TCA"; EgXTH1 (accession no. AB762070), "TGC ATT GAA TTG AAC ATC GAA" and "CAT GGT TCC ACA AAC AAA TCC". Relative gene expression levels were calculated using the comparative $\mathrm{Ct}$ method, including normalization against constitutively expressed ubiquitin (EgUbi: GenBank, AB049409.1) and against a control sample (petals at stage IV). The primer pair for EgUbi was "ATCCAGTGACACCATCGACA" and "TCCATC CTCCAACTGCTTTC".

\section{Antibody development}

An antibody was raised against recombinant EgEXPA1 or EgXTH1. EgEXPAl or EgXTH1 cDNA were cloned into pCOLD FT (Takara) containing the histidine-tag sequence and then each plasmid was transformed into Escherichia coli BL21 (DE3). The cultures were developed for $24 \mathrm{~h}$ at $15^{\circ} \mathrm{C}$ with IPGT at a final concentration of $1 \mathrm{mM}$ after incubation for $12 \mathrm{~h}$ at $37^{\circ} \mathrm{C}$. The cells were centrifuged at $3000 \times \mathrm{g}$ for $10 \mathrm{~min}$. The recombinant proteins were purified by TALON Metal Affinity Resins (Clontech Laboratories, Palo Alto, CA, USA) from pellets. Purified proteins were used as antigens for guinea pig inoculation. Antibodies were purified by immunoaffinity from immune sera. Recombinant EgEXPA1 or EgXTH1 expressed by pET32 (Novagen) in E. coli BL21 (DE3) was used as affinity ligands for each antibody.

\section{Protein extraction}

Frozen petals were homogenized in $10 \mathrm{mM}$ sodium phosphate buffer (pH 6.0) containing $1 \mathrm{M}$ sodium chloride and $1 \%(\mathrm{v} / \mathrm{v})$ glycerol. After centrifugation at $20000 \times \mathrm{g}$ for $10 \mathrm{~min}, 80 \%(\mathrm{w} / \mathrm{v})$ ammonium sulphate was added to the supernatant and kept at $4{ }^{\circ} \mathrm{C}$ overnight. The mixture was centrifuged at $20000 \times \mathrm{g}$ for $20 \mathrm{~min}$, and the pellet was dissolved in $8 \mathrm{M}$ urea. Protein amount was determined by Bio-Rad Protein Assay (BIO-RAD, Hercules, CA, USA).

\section{Western blotting analysis}

Protein solution containing $20 \mu \mathrm{g}$ protein was separated by SDS-PAGE using $12 \%(\mathrm{w} / \mathrm{v})$ polyacrylamide gel and electrotransfered to Hybond-P (GE Healthcare, Little Chalfont, UK). The protein was probed with an antibody raised against recombinant EgEXPA1 or EgXTH1 followed by a horseradish peroxidaseconjugated secondary antibody. The blot was detected with ECL Prime Western Blotting Detection System (GE Healthcare).

\section{Results}

\section{Eustoma petal growth}

Eustoma petals at the following 6 stages were used for experiments, as shown in Figure 1A. Petals curved outward in the upper and middle parts from stage IV to VI, concurrently with coloration of petals, resulting in flower opening. Vertical elongation of petals occurred throughout all stages. The change in the fresh weight of petals and the time to reach each stage from stage I are shown in Figure 1B. Although an increase of petal fresh weight was confirmed at all stages, a rapid increase occurred only in later stages. Petal growth in later stages was apparently more marked than that at early stages. The most dynamic change of petals was observed from stage IV to VI in fresh weight, shape, and color. Extensibility of petals at each flower stage is shown in Figure 2. No change in extensibility was observed between stage II and III. A constant increase of extensibility was seen during stage III to VI. This increase of extensibility was coincident with a marked change in fresh weight and appearance of petals.

\section{Cloning of expansins and XTH cDNAs from Eustoma petals}

Three and one distinct putative cDNAs of $\alpha$-expansin and $X T H$, respectively, were isolated from opening Eustoma petals by RT-PCR. $\alpha$-Expansin cDNAs were named EgEXPA1, EgEXPA2, and EgEXPA3, and an XTH cDNA $E g X T H 1$. The full-length sequences of these cDNAs were obtained by 5'RACE. The predicted proteins of EgEXPA1, EgEXPA2, EgEXPA3, and EgXTH1 were composed of 258, 262, 249, and 295 amino acids, respectively. All of the 3 deduced amino acid sequences of expansin had 8 cysteine residues, 4 tryptophan residues and an HFD domain, which were

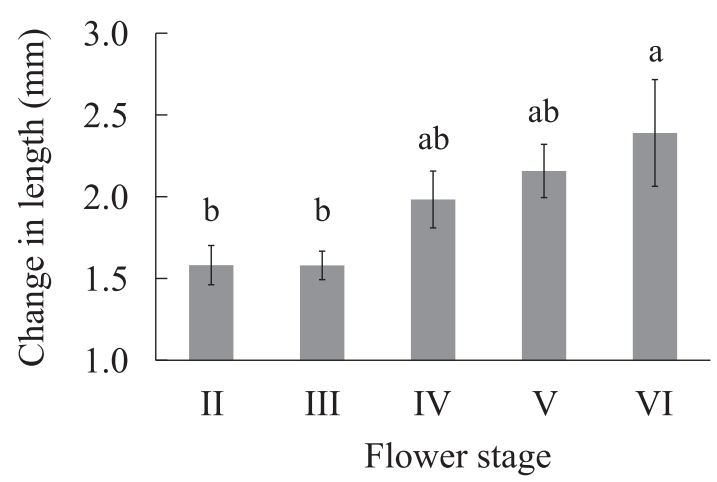

Fig. 2. Extensibility of petals at each flower stage. Petal strips were secured between 2 clamps positioned $5 \mathrm{~mm}$ apart and extended at constant $10 \mathrm{~g}$ weight. The distance between clamps was recorded $30 \mathrm{~min}$ after starting. Each value represents the mean $\pm \mathrm{SE}(\mathrm{n}=4$ or 6$)$. Different letters indicate that the values were significantly different according to Tukey's multiple range test at $P<0.05$. 


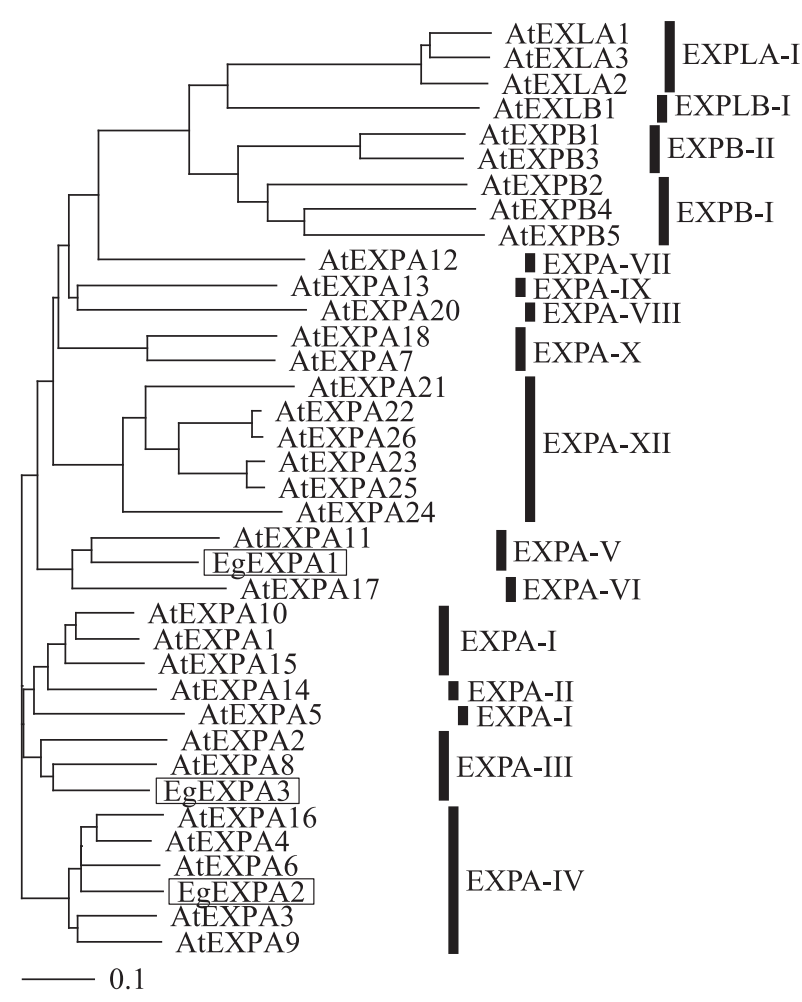

Fig. 3. Phylogeny of Eustoma and Arabidopsis thaliana expansins using unweighted pair group method with arithmetic mean Genes newly identified from Eustoma in this study are shown in boxes.

highly conserved motifs in Arabidopsis thaliana expansins, in corresponding regions (Li et al., 2003). They had an $\alpha$-insertion and no $\beta$-insertion (data not shown). The EgXTH1 predicted protein had the DEIDFEFLG domain, N-linked glycosylation site and 4 cysteine residues. According to phylogenetic analysis with Arabidopsis expansins and XTHs, EgEXPA1, EgEXPA2, and EgEXPA3 belong to clades EXPA-V, IV, and III, respectively (Fig. 3). EgXTH1 belongs to Group 1 of XTH family (Fig. 4).

\section{EgEXPA1-3 and EgXTH1 expression patterns among different organs and during petal growth}

The transcripts levels of EgEXPA1-3 and EgXTHI were analyzed in mature leaves, young leaves, stems, roots, sepals, stamens, pistils, and petals at each flower stage by quantitative real-time RT-PCR (Fig. 5). They showed abundant expression in petals and no or little expression in other organs. Thus, the expression of all 4 genes showed more or less specificity to the petals. Focusing on petals, whereas all genes evidenced low expression at early stages, their expression increased largely around stage III and they exhibited marked expression at later stages. The expression of EgEXPAI and 2 peaked at stage IV. EgEXPA3 and EgXTHI marked the highest expression at stage $\mathrm{V}$. The period between stage IV and $\mathrm{V}$ was the period when just the petals were bending outwards.

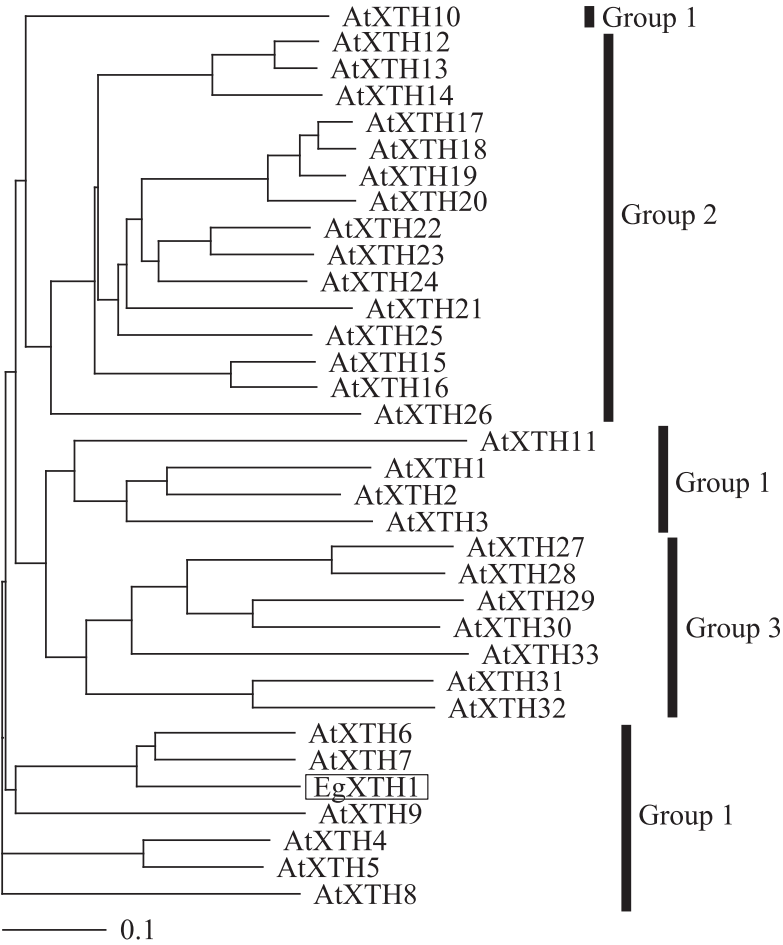

Fig. 4. Phylogeny of Eustoma and Arabidopsis thaliana XTHs using unweighted pair group method with arithmetic mean. A gene newly identified from Eustoma in this study is shown in a box.

\section{Accumulation of Expansin and XTH proteins during petal growth}

Accumulation of expansin and XTH proteins in petals was analyzed by Western blotting. Antibodies were raised against recombinant EgEXPA1 and EgXTH1. Both antibodies detected the protein with unique molecular size resembling crude expansins or XTHs. During stages I and II, no accumulation of expansin protein was observed, but abundant accumulation was detected from stages III to VI (Fig. 6A). Accumulation increased as the flower stages progressed. XTH protein showed no detectable accumulation from stage I to stage III; however, the expression of XTH protein started increasing during stages $\mathrm{IV}$ and $\mathrm{V}$ and exhibited extensive accumulation from stage $\mathrm{V}$ and VI.

\section{Discussion}

After stage IV, marked changes were seen in fresh weight, growth rate, extensibility, shape, and color (Figs. 1 and 2). These rapid changes occurred simultaneously and resulted in flower opening. In contrast, until stage III, fresh weight increased moderately but extensibility did not. Thus, unveiling the mechanism of petal growth coordinating with cell loosening is the key to controlling flower opening. It is known that expansins and XTHs participate in cell loosening in many plants, and have been identified from petals in some plants. Likewise, some expansins and XTHs have important roles in flower opening by increasing petal cell-wall extensibility in Eustoma. 

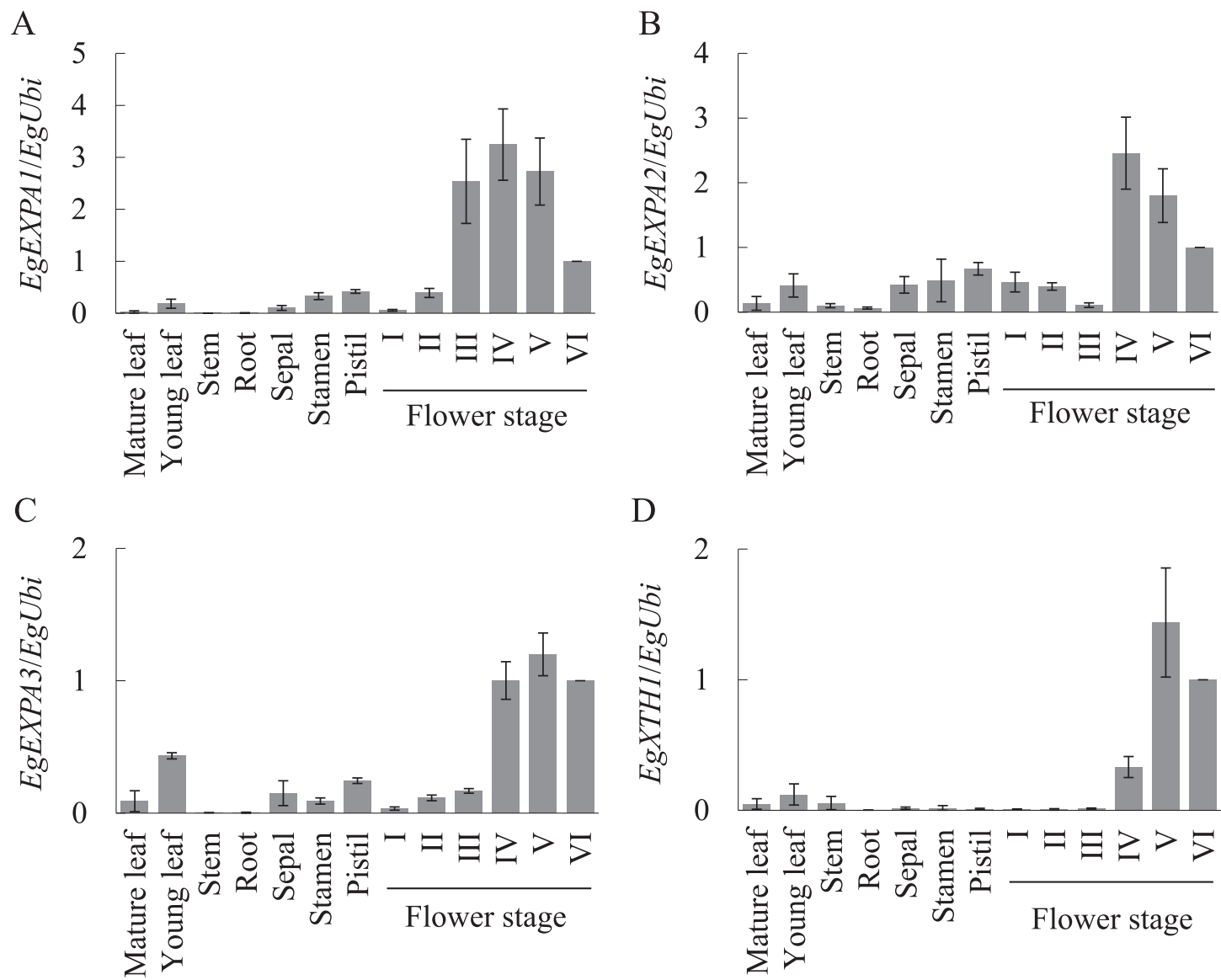

Fig. 5. The relative levels of expansin and XTH mRNAs in various organs. EgEXPA1 (A), EgEXPA2 (B), EgEXPA3 (C), and EgXTH1 (D). Expression levels were normalized against $E g U b i$ as an internal control and expressed as a ratio against the value for petals at stage VI. Each value is the mean \pm SE (petals; $n=6$, other organs; $n=3$ ).

A

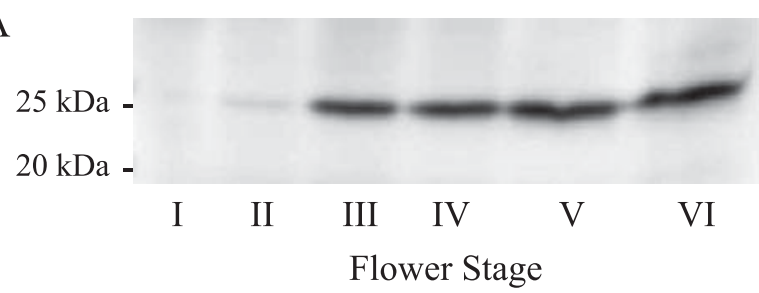

B

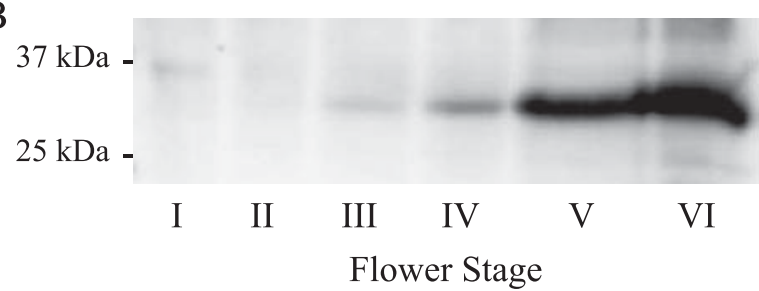

Fig. 6. Expression profiles of expansin and XTH proteins in petals. Twenty micrograms of extracted proteins from petals were subjected to SDS-PAGE and Western blot using antibody raised to EgEXPA1 (A) or EgXTH1 (B). Positions of molecular mass standards are shown on the left.

We obtained three distinct putative expansin cDNAs and one putative $X T H$ cDNA, which were named EgEXPA1, EgEXPA2, EgEXPA3, and EgXTH1, respectively, from opening Eustoma petals by RT-PCR.
EgEXPA1, EgEXPA2, and EgEXPA3 are certainly $\alpha$ expansins, which are thought to be separated into 12 clades. EgEXPA1, EgEXPA2, and EgEXPA3 belong to clades EXPA-V, IV, and III, respectively (Fig. 3). Clade EXPA-V, which includes EgEXPA1, contains most rice $\alpha$-expansins but only one Arabidopsis $\alpha$-expansin, AtEXPA11. The function of $\alpha$-expansins belonging to clade EXPA-V in dicot plants remains unclear. It has been proposed that expansins belonging to clade EXPAIV may play an important role in xylem development (Sampedro et al., 2006). Thus, EgEXPA2 may be involved in xylem development. Clade EXPA-III, which includes EgEXPA3, contains many $\alpha$-expansin genes that are expressed in rapidly growing tissue. For example, CsExp1, LeExp2, and OsExp4 (Catalá et al., 2000; Shcherban et al., 1995) are expressed in elongating hypocotyls. This suggests that EgEXPA3 may relate primarily to petal growth. XTHs are thought to be separated into 3 clades. According to phylogenetic analysis with Arabidopsis XTHs, EgXTH1 belongs to Group 1 of the XTH family (Fig. 4). Members of Groups 1 and 2 are believed to have XET (xyloglucan endotransglucosylase) activity that catalyses both cleavage and relegation, while members of Groups 3 are believed to have XEH (xyloglucan endohydrolyse) 
activity that catalyses only cleavage (Rose et al., 2002). This suggests that EgXTH1 belonging to Group 1 may have mediate transglucosylation between xyloglucans.

The transcription levels of EgEXPA1-3 and EgXTH1 were analyzed in petals at each stage and other organs by quantitative real-time RT-PCR (Fig. 5). All 4 genes exhibited distinct expression in petals, in contrast to other floral and vegetative organs, and these should be the genes mainly taking part in petal cell growth in Eustoma. Petal growth in Eustoma can be divided into 2 steps. First, petal growth unaccompanied by changes in cell-wall extensibility seen from stages I to III, and second, petal growth accompanied by increased cell-wall extensibility (Fig. 2). All 4 gene transcripts were abundant in later stages but not in early stages. Hence, EgEXPA1-3 and EgXTH1 participate in the petal growth accompanying the increase in cell-wall extensibility. This growth pattern results in flower opening; therefore, EgEXPA1-3 and EgXTH1 are involved in petal growth during flower opening. In the early stages when EgEXPA1-3 and EgXTH1 showed little expression, petal growth is thought to occur according to the changes in petal size and fresh weight without any petal extensibility change. There are some reports that some expansin and $X T H$ genes were expressed in the abscission zone of petals when flowers senesced (Sane et al., 2007; Singh et al., 2011); however, the transcription levels of EgEXPA1-3 and EgXTH1 kept decreasing after stage V when the flowers continued opening. Thus, they might not be involved in petal growth in bud stages or petal senescence. During flower growth, the expression of EgEXPA2 and EgEXPA3 increased abundantly from stages III to IV, the period when flowers are about to open. The expression of EgXTH1 increased when the upper part of petals was just bending outwards, and the expression of EgEXPA1 increased markedly from stages II to III, ahead of flower opening. Considering the phylogenetic analyses, EgEXPA3 may be a major contributor to the rapid petal growth resulting in flower opening in Eustoma. In roses, it was shown that several expansins and XTHs took part in flower opening, and the expression regions of these genes were different in parts or stages of petals (Yamada et al., 2009). Thus, it is possible that all genes cooperatively take part in flower opening or specialize in parts of petals on the proximaldistal axis or adaxial-abaxial axis.

Accumulation of expansins and XTHs protein in petals was analyzed by Western blotting. Both antibodies raised against recombinant EgEXPA1 and EgXTH1 could detect specific proteins with molecular masses of about 25 or $30 \mathrm{kDa}$, respectively (Fig. $6 \mathrm{~A}, \mathrm{~B}$ ). The deduced molecular masses of EgEXPA1, 2, 3, and EgXTH1 excluding deduced signal peptides were 25.4, 26.1, 24.3, and $30.1 \mathrm{kDa}$, respectively. These antibodies could also detect each recombinant protein constructed with a vector different from that used for antigens (data not shown). Thus, they should be able to detect whole expansin and XTH proteins extracted from petals.

Abundant accumulation of expansin proteins was detected from stages III to VI (Fig. 6A), although apparent accumulation was not observed during stages I and II. The accumulation dosage increased as the flower stages progressed. This trend was coincident with the changes in petal fresh weight and extensibility. This trend was also similar to the combination of expression patterns of EgEXPA1, 2, and 3 cDNAs, especially in the early and middle stages. In stage VI, however, the amount of expansin protein was still high, even though the expression of all 3 expansins decreased. This was likely due to the duration of protein synthesis and degradation, or perhaps the accumulation of other expansins related to petal senescence. The remaining expansin protein may induce the petal growth in stage VI. XTH protein showed no detectable accumulation from stage I to stage III; however, the expression of XTH protein increased at stage IV and accumulated extensively at stages $\mathrm{V}$ and VI. This accumulation pattern highly resembled the EgXTH1 mRNA expression pattern. The different expression patterns between $E g X T H 1$ cDNA and XTH protein after stage $\mathrm{V}$ were similar to those of expansins. EgXTH1 could contribute to partial petal growth when the upper parts of petals are just bending outwards, neither in bud stages nor the timing of bud bursting.

These results suggest that EgEXPA1-3 and EgXTH1 exclusively correlated to flower opening in Eustoma. More particularly, EgEXPA1-3 were continuously involved in petal growth from bud (stage III) to flower opening (stage VI) and EgXTH1 played a role in rapid petal growth accompanied by dynamic changes in petal fresh weight and petal shape from stage IV to stage VI.

These results are consistent with a previous study that showed a correlation between expansin and XTH mRNA expression levels and rose flower opening (Takahashi et al., 2007). In this research, we showed the contribution of expansin and XTH to flower opening in more depth by unveiling the trends of these protein accumulations using Western blot analysis. We are now analyzing the petal phenotype using transgenic Eustomas in which expansins or XTHs are knocked down. This work will explore the roles that expansins and XTHs play in regulating petal cell-wall extensibility and petal growth. It is worthwhile trying to examine the effect of inhibitors, antibodies and recombinant proteins on the petal growth of cut flowers under postharvest conditions with the aim of improving cut flower quality and the appreciation period. The speed of flower opening could be controlled under postharvest conditions, if we could regulate the activity of these proteins.

\section{Literature Cited}

Adams, P. A., M. J. Montague, M. Tepfer, D. L. Rayle, H. Ikuma and P. B. Kaufman. 1975. Effect of gibberellic acid on the plasticity and elasticity of Avena stem segments. Plant Physiol. 56: 757-760. 
Belfield, E. J., B. Ruperti, J. A. Roberts and S. J. McQueen-Mason. 2005. Changes in expansin activity and gene expression during ethylene-promoted leaflet abscission in Sambucus nigra. J. Exp. Bot. 56: 817-823.

Brummel, D. A., M. H. Harpster and P. Dunsmuir. 1999. Differential expression of expansin gene family members during growth and ripening of tomato fruit. Plant Mol. Biol. 39: 161-169.

Carpita, N. C. and J. Kanabus. 1988. Chemical structure of the cell walls of dwarf maize and changes mediated by gibberellin. Plant Physiol. 88: 671-678.

Catalá, C., J. K. C. Rose and A. B. Bennett. 2000. Auxin-regulated genes encoding cell wall-modifying proteins are expressed during early tomato fruit growth. Plant Physiol. 122: 527-534.

Civello, P. M., A. L. T. Powell, A. Sabehat and A. B. Bennett. 1999. An expansin gene expressed in ripening strawberry fruit. Plant Physiol. 121: 1273-1279.

Cosgrove, D. J. 2000. Expansive growth of plant cell walls. Plant Physiol. Biochem. 38: 109-124.

Cosgrove, D. J. 2001. Wall structure and wall loosening. A look backwards and forwards. Plant Physiol. 125: 131-134.

de Vetten, N. C. and D. J. Huber. 1990. Cell wall changes during the expansion and senescence of carnation (Dianthus caryophyllus) petals. Physiol. Plant. 78: 447-454.

Evans, R. Y. and M. S. Reid. 1988. Changes in carbohydrates and osmotic potential during rhythmic expansion of rose petals. J. Amer. Soc. Hort. Sci. 113: 884-888.

Gookin, T. E., D. A. Hunter and M. S. Reid. 2003. Temporal analysis of alpha and beta-expansin expression during floral opening and senescence. Plant Sci. 164: 769-781.

Harada, T., Y. Torii, S. Morita, R. Onodera, Y. Hara, R. Yokoyama, K. Nishitani and S. Satoh. 2010. Cloning, characterization, and expression of xyloglucan endotransglucosylase/hydrolase and expansin genes associated with petal growth and development during carnation flower opening. J. Exp. Bot. 62: 815-823.

Ho, L. C. and R. Nichols. 1977. Translocation of ${ }^{14} \mathrm{C}$-sucrose in relation to changes in carbohydrate content in rose corollas cut at different stages of development. Ann. Bot. 41: 227-242.

Hohl, M. and P. Schopfer. 1992. Growth at reduced turgor: irreversible and reversible cell-wall extension of maize coleoptiles and its implications for the theory of cell growth. Planta 187: 209-217.

Ichimura, K., Y. Kawabata, M. Kishimoto, R. Goto and K. Yamada. 2003. Shortage of soluble carbohydrates is largely responsible for short vase life of cut 'Sonia' rose flowers. J. Japan. Soc. Hort. Sci. 72: 292-298.

Li, Y., Jones L. and S. J. McQueen-Mason. 2003. Expansins and cell growth. Curr. Opin. Plant Biol. 6: 603-610.

McQueen-Mason, S. J., D. M. Durachko and D. J. Cosgrove. 1992. Two endogenous proteins that induce cell-wall extension in plants. Plant Cell 4: 1425-1433.

Matsukura, C., S. Itoh, K. Nemoto, E. Tanimoto and J. Yamaguchi. 1998. Promotion of leaf sheath growth by gibberellic acid in a dwarf mutant of rice. Planta 205: 145-152.

Nakamura, T., S. Sekine, K. Arai and N. Takahashi. 1975. Effects of gibberellic acid and indole-3-acetic acid on stress relaxation properties of pea hook cell wall. Plant Cell Physiol. 16: 127138.

Nishitani, K. and R. Tominaga. 1992. Endo-xyloglucan transferase, a novel class of glycosyltransferase that catalyzes transfer of a segment of xyloglucan molecule to another xyloglucan molecule. J. Biol. Chem. 267: 21058-21064.

O'Donoghue, E. M., S. D. Somerfield and J. A. Heyes. 2002.
Organization of cell walls in Sandersonia aurantiaca floral tissue. J. Exp. Bot. 53: 513-523.

Okazawa, K., Y. Sato, T. Nakagawa, K. Asada, I. Kato, E. Tomita and K. Nishitani. 1993. Molecular cloning and cDNA sequencing of endoxyloglucan transferase, a novel class of glycosyltransferase that mediates molecular grafting between matrix polysaccharides in plant cell walls. J. Biol. Chem. 268: 25364-25368.

Rose, J. K. C. and A. B. Bennett. 1999. Cooperative disassembly of the cellulose-xyloglucan network of plant cell walls: parallels between cell expansion and fruit ripening. Trends Plant Sci. 4: 176-183.

Rose, J. K. C., J. Braam, S. C. Fry and K. Nishitani. 2002. The XTH family of enzymes involved in xyloglucan endotransglucosylation and endohydrolysis: current perspectives and a new unifying nomenclature. Plant Cell Physiol. 43: 14211435.

Rose, J. K. C., H. H. Lee and A. Bennett. 1997. Expression of a divergent expansin gene is fruit-specific and ripeningregulated. Proc. Natl. Acad. Sci. USA 94: 5955-5960.

Sampedro, J., R. E. Carey and D. J. Cosgrove. 2006. Genome histories clarify evolution of expansin superfamily: new insights from the poplar genome and pine ESTs. J. Plant Res. 119: 11-21.

Sane, A. P., S. K. Tripathi and P. Nath. 2007. Petal abscission in rose (Rosa bourboniana var Gruss an Teplitz) is associated with the enhanced expression of an alpha expansin gene, RbEXPA1. Plant Sci. 172: 481-487.

Shcherban, T. Y., J. Shi, D. M. Durachko, M. J. Guiltinan, S. J. McQueen-Mason, M. Shieh and D. J. Cosgrove. 1995. Molecular cloning and sequence analysis of expansins: a highly conserved, multigene family of proteins that mediate cell wall extension in plants. Proc. Natl. Acad. Sci. USA 92: 9245-9249.

Singh, A., S. Tripathi, P. Nath and A. Sane. 2011. Petal abscission in rose is associated with the differential expression of two ethylene-responsive xyloglucan endotransglucosylase/hydrolase genes, RbXTH1, and RbXTH2. J. Exp. Bot. 62: 5091-5103.

Takahashi, R., C. Fujitani, S. Yamaki and K. Yamada. 2007. Analysis of the cell wall loosening proteins during rose flower opening. Acta Hort. 755: 483-488.

Tanimoto, E. and Y. Masuda. 1971. Role of the epidermis in auxininduced elongation of light-grown pea stem segments. Plant Cell Physiol. 12: 663-673.

Tanimoto, E., S. Fujii, R. Yamamoto and S. Inanaga. 2000. Measurement of viscoelastic properties of root cell walls affected by low $\mathrm{pH}$ in lateral roots of Pisum sativum L. Plant Soil 226: 21-28.

van Doorn, W. G. and E. J. Woltering. 2008. Physiology and molecular biology of petal senescence. J. Exp. Bot. 59: 453480.

Yamada, K., R. Takahashi, C. Fujitani, K. Mishima, M. Yoshida, D. C. Joyce and S. Yamaki. 2009. Cell wall extensibility and effect of cell-wall-loosening proteins during rose flower opening. J. Japan. Soc. Hort. Sci. 78: 242-251.

Yamamoto, R., K. Shimizu and Y. Masuda. 1970. Stress-relaxation properties of plant cell walls with special reference to auxin action. Plant Cell Physiol. 11: 947-956.

Zenoni, S., L. Reale, G. B. Tornielli, L. Lanfaloni, A. Porceddu, A. Ferrarini, C. Moretti, A. Zamboni, A. Speghini, F. Ferranti and M. Pezzotti. 2004. Downregulation of the Petunia hybrida $\alpha$-expansin gene PhEXP1 reduces the amount of crystalline cellulose in cell walls and leads to phenotypic changes in petal limbs. Plant Cell 16: 295-308. 